\title{
Design of rectangular microstrip antenna with rectangular aperture in the ground plane using artificial neural networks
}

\author{
Siham Benkouda \\ Electronics Department \\ University of Frères Mentouri - Constantine 1 \\ Constantine, Algeria \\ s_benkouda@yahoo.fr \\ Tarek Fortaki1, Sami Bedral, and Abdelkrim Belhedri2 \\ Electronics Department \\ ${ }^{1}$ University of Batna \\ ${ }^{2}$ University of Frères Mentouri - Constantine 1 \\ Algeria \\ t_fortaki@yahoo.fr
}

\begin{abstract}
In this paper, we propose a general design of rectangular microstrip antenna with and without rectangular aperture over ground plane, based on artificial neural networks (ANN) in conjunction with spectral domain formulation. In the design procedure, syntheses ANN model is used as feed forward network to determine the resonant frequency and bandwidth. Analysis ANN model is used as the reversed of the problem to calculate the antenna and aperture dimensions for the given resonant frequency, dielectric constant and height of substrate. The spectral domain formulation combined with artificial neural network in the analysis and the design of rectangular antenna to reduce the complexity of the spectral approach and to minimize the CPU time necessary to obtain the numerical results. The results obtained from the neural models are in very good agreement with the experimental results available in the literature.
\end{abstract}

Keywords-microstrip antenna; artificial neural network; modeling

\section{INTRODUCTION}

Microstrip antennas (MSAs) are used in a broad range of applications from communication systems to biomedical systems, primarily due to their simplicity, conformability, low manufacturing cost, light weight, low profile, reproducibility, reliability, and ease in fabrication and integration with solidstate devices [1-2]. The main shortcomings of these antennas are narrow bandwidth and low gain. These shortcomings can be overcome in by proper design of an antenna, and especially by using proper substrate thickness and dielectric constant as well as a proper way of feeding [3-5].

Several methods [6-9], varying in accuracy and computational effort, have been proposed and used to calculate the resonant characteristics of various microstrip antennas shapes. Generally, there are two methods for analysis of microstrip antenna such as numerical method and analytical method. Despite simple analytical methods giving a good intuitive explanation of antenna radiation properties, exact mathematical formulations involve extensive numerical procedures, resulting in round-off errors and possibly needing final experimental adjustments to the theoretical results [2]. The numerical methods are complicated compared to analytical methods [10]. They are also time consuming and not easily included in a computer-aided design package [1-2]. On the other hand, commercial software uses computerintensive numerical methods such as, finite element method (FEM), method of moment (MoM), finite difference time domain (FDTD) method, etc.... But the resulting codes are often too slow for design purposes, since they take a lot of computation time and require large computer resources [11]. To reach to a final optimized structure, it might need several simulations. In order to reduce this time of computation, some commercially available packages are now available with optimizers, but for this also, number of simulations are required [11]. It is well-known that the electromagnetic simulation takes tremendous computational efforts, and the practical measurement is expensive [12]

Currently, computer-aided design (CAD) models based on artificial neural networks (ANNs) have been applied for analysis and synthesis of microstrip antennas in various forms such as rectangular, square, and circular patch antennas [13]. Due to their ability and adaptability to learn, generalizability, smaller information requirement, fast real-time operation, and ease of implementation features [1], neural network models are used extensively for wireless communication engineering, which eliminate the complex and time-consuming mathematical procedure of designing, like the method of 
moments [14]. The neural networks in conjunction with spectral domain approach was firstly proposed by Mishra and Patnaik [15], to calculate the complex resonant frequency and the input impedance [16] of rectangular microstrip antenna, this approach is named neurospectral method [8]. This is the main reason for selecting the neurospectral to estimate the resonant frequency and half-power bandwidth of a rectangular microstrip patch over ground plane with rectangular aperture. The analysis model is used to obtain the resonant frequency for a given dielectric material and patch structure, whereas the synthesis model is built to determine patch and aperture dimensions for the required design specifications [12].

The objective of this work is to present an integrated approach based on artificial neural networks and spectral domain approach. We introduce the artificial neural networks in the analysis and synthesis of a rectangular microstrip patch over a ground plane with rectangular aperture to reduce the complexity of the spectral approach and to minimize the CPU time necessary to obtain the numerical results. The neurospectral model is simple, easy to apply, and very useful for antenna engineers to predict both resonant frequency and half-power bandwidth.

\section{SPECTRAL DOMAIN FORMULATION}

The geometry of the considered structure is shown in Fig.1. We have a rectangular microstrip patch of length $L_{p}$ along the $x$ direction and width $W_{p}$ along y direction over ground plane with a rectangular aperture of length $L_{a}$ and width $W_{a}$. Both the center of the patch and the center of aperture have the coordinate value $(x, y)=(0,0)$. Also, the metallic patch and the ground plane are assumed to be perfect electric conductors of negligible thickness. The dielectric layer of thickness $d$ is characterized by the free-space permeability $\mu_{0}$ and the permittivity $\varepsilon_{0}, \varepsilon_{r}\left(\varepsilon_{0}\right.$ is the free-space permittivity and the relative permittivity $\varepsilon_{r}$ can be complex to account for dielectric loss). The ambient medium is air with constitutive parameters $\mu_{0}$ and $\varepsilon_{0}$.

All fields and currents are time harmonic with the $\varepsilon^{i \omega t}$ time dependence suppressed. The transverse fields inside the substrate region can be obtained via the inverse vector Fourier transforms as $[4,17]$

$$
\begin{gathered}
\mathbf{E}\left(\mathbf{r}_{s}, z\right)=\left[\begin{array}{c}
E_{x}\left(\mathbf{r}_{s}, z\right) \\
E_{y}\left(\mathbf{r}_{s}, z\right)
\end{array}\right]=\frac{1}{4 \pi^{2}} \int_{-\infty}^{+\infty+\infty} \int_{-\infty}^{\infty} \overline{\mathbf{F}}\left(\mathbf{k}_{s}, \mathbf{r}_{s}\right) \cdot \mathbf{e}\left(\mathbf{k}_{s}, z\right) d k_{x} d k_{y}(1) \\
\mathbf{H}\left(\mathbf{r}_{s}, z\right)=\left[\begin{array}{c}
H_{y}\left(\mathbf{r}_{s}, z\right) \\
-H_{x}\left(\mathbf{r}_{s}, z\right)
\end{array}\right]=\frac{1}{4 \pi^{2}} \int_{-\infty}^{+\infty+\infty} \int_{-\infty}^{\infty} \overline{\mathbf{F}}\left(\mathbf{k}_{s}, \mathbf{r}_{s}\right) \cdot \mathbf{h}\left(\mathbf{k}_{s}, z\right) d k_{x} d k_{y}(2)
\end{gathered}
$$

where $\overline{\mathbf{F}}\left(\mathbf{k}_{s}, \mathbf{r}_{s}\right)$ is the kernel of the vector Fourier transforming domain (VFTD) [4, 17]

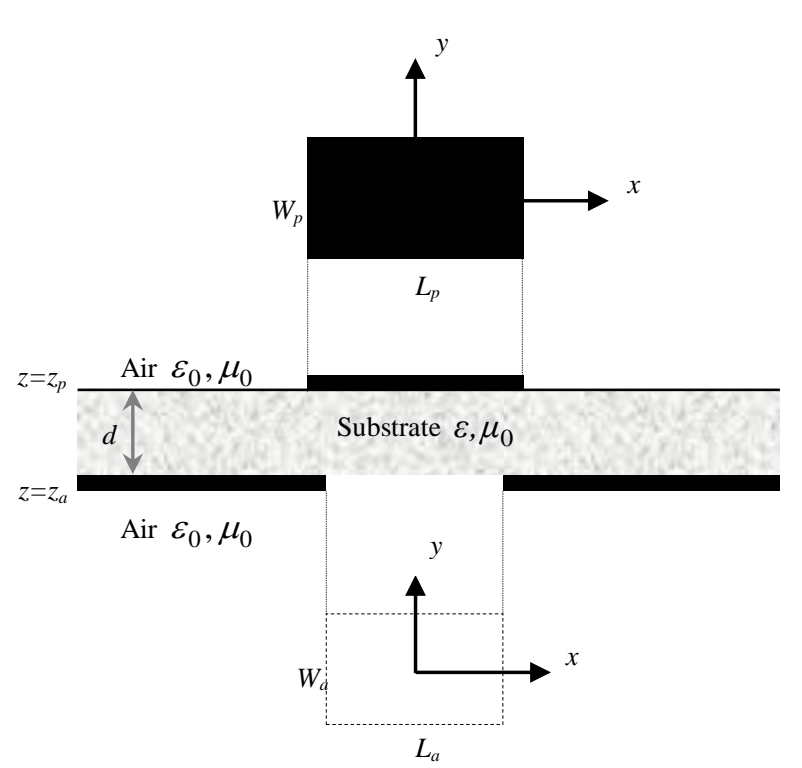

Figure 1. Geometrical structure of a tunable rectangular microstrip patch over a ground plane with rectangular aperture.

$$
\begin{aligned}
& \overline{\mathbf{F}}\left(\mathbf{k}_{s}, \mathbf{r}_{s}\right)=\frac{1}{k_{s}} \cdot\left[\begin{array}{cc}
k_{\mathrm{x}} & k_{\mathrm{y}} \\
k_{\mathrm{y}} & -k_{\mathrm{x}}
\end{array}\right] \cdot \mathrm{e}^{\mathrm{i} \mathbf{k}_{s} \cdot \mathbf{r}_{s}}, \\
& \mathbf{r}_{s}=\hat{\mathbf{x}} x+\hat{\mathbf{y}} y, \mathbf{k}_{s}=\hat{\mathbf{x}} k_{x}+\hat{\mathbf{y}} k_{y}, k_{s}=\left|\mathbf{k}_{s}\right|
\end{aligned}
$$

The relation witch related the current $\mathbf{j}\left(\mathbf{k}_{s}\right), \mathbf{j}_{0}\left(\mathbf{k}_{s}\right)$ on the conducting patch (ground plane with rectangular aperture) to the electric field on the corresponding interface $\mathbf{e}\left(\mathbf{k}_{s}, z_{p}\right)$, and $\mathbf{e}\left(\mathbf{k}_{s}, z_{a}\right)$ given by

$$
\begin{gathered}
\mathbf{e}\left(\mathbf{k}_{s}, z_{p}\right)=\overline{\mathbf{G}}\left(\mathbf{k}_{s}\right) \cdot \mathbf{j}\left(\mathbf{k}_{s}\right)+\overline{\mathbf{\Psi}}\left(\mathbf{k}_{s}\right) \cdot \mathbf{e}\left(\mathbf{k}_{s}, z_{\mathrm{a}}\right) \\
\mathbf{j}_{0}\left(\mathbf{k}_{s}\right)=-\overline{\boldsymbol{\Phi}}\left(\mathbf{k}_{s}\right) \cdot \mathbf{j}\left(\mathbf{k}_{s}\right)-\overline{\mathbf{Y}}\left(\mathbf{k}_{s}\right) \cdot \mathbf{e}\left(\mathbf{k}_{s}, 0\right)
\end{gathered}
$$

The four $2 \times 2$ diagonal matrices $\overline{\mathbf{G}}\left(\mathbf{k}_{s}\right), \overline{\mathbf{\Psi}}\left(\mathbf{k}_{s}\right), \overline{\mathbf{\Phi}}\left(\mathbf{k}_{s}\right)$, and $\overline{\mathbf{Y}}\left(\mathbf{k}_{s}\right)$ stand for a set of dyadic Green's functions in the vector Fourier transform domain. It is to be noted that $\overline{\mathbf{G}}\left(\mathbf{k}_{s}\right)$ is related to the patch current and $\overline{\mathbf{Y}}\left(\mathbf{k}_{s}\right)$ is related to the aperture field. $\overline{\boldsymbol{\Psi}}\left(\mathbf{k}_{s}\right)$ and $\overline{\boldsymbol{\Phi}}\left(\mathbf{k}_{s}\right)$ represent the interactions between the patch current and aperture field. In Equations (4) and (5) the unknowns are $\mathbf{j}\left(\mathbf{k}_{s}\right)$ and $\mathbf{e}\left(\mathbf{k}_{s}, z_{a}\right)$. Another possible choice in the analysis of microstrip patches over ground planes with apertures is to consider $\mathbf{j}_{0}\left(\mathbf{k}_{s}\right)$ as unknown instead of $\mathbf{e}\left(\mathbf{k}_{s}, z_{a}\right)$. It is anticipated, however, that a very large number of terms of basis functions would be 
needed for the expansion of the current $\mathbf{j}_{0}\left(\mathbf{r}_{s}\right)$ on the ground plane with aperture because of the wide conductor area. Hence, it is better to apply the Galerkin procedure to the unknown $\mathbf{E}\left(\mathbf{r}_{s}, z_{a}\right)$ field at the aperture [17].

The transverse electric field at the plane of the patch and the surface current density on the ground plane with a rectangular aperture can be obtained from Equations (4) and (5), respectively, via the inverse vector Fourier transforms as

$$
\begin{aligned}
& \mathbf{E}\left(\mathbf{r}_{s}, z_{p}\right)=\frac{1}{4 \pi^{2}} \int_{-\infty}^{+\infty} \int_{-\infty}^{+\infty} \overline{\mathbf{F}}\left(\mathbf{k}_{s}, \mathbf{r}_{s}\right) \cdot \\
& {\left[\overline{\mathbf{G}}\left(\mathbf{k}_{s}\right) \cdot \mathbf{j}\left(\mathbf{k}_{s}\right)+\overline{\mathbf{\Psi}}\left(\mathbf{k}_{s}\right) \cdot \mathbf{e}\left(\mathbf{k}_{s}, z_{a}\right)\right] d k_{x} d k_{y}} \\
& \mathbf{J}_{0}\left(\mathbf{r}_{s}\right)=-\frac{1}{4 \pi^{2}} \int_{-\infty}^{+\infty} \int_{-\infty}^{+\infty} \overline{\mathbf{F}}\left(\mathbf{k}_{s}, \mathbf{r}_{s}\right) . \\
& {\left[\overline{\mathbf{\Phi}}\left(\mathbf{k}_{s}\right) \cdot \mathbf{j}\left(\mathbf{k}_{s}\right)+\overline{\mathbf{Y}}\left(\mathbf{k}_{s}\right) \cdot \mathbf{e}\left(\mathbf{k}_{s}, z_{a}\right)\right] d k_{x} d k_{y}}
\end{aligned}
$$

Boundary conditions require that the transverse electric field of Equation (6) vanishes on the perfectly conducting patch and the current of Equation (7) vanishes off the ground plane, to give the following coupled integral equations for the patch current and aperture field:

$$
\begin{array}{r}
\int_{-\infty}^{+\infty} \int_{-\infty}^{+\infty} \overline{\mathbf{F}}\left(\mathbf{k}_{s}, \mathbf{r}_{s}\right) \cdot\left(\overline{\mathbf{G}}\left(\mathbf{k}_{s}\right) \cdot \mathbf{j}\left(\mathbf{k}_{s}\right)+\overline{\mathbf{\Psi}}\left(\mathbf{k}_{s}\right) \cdot\right. \\
\left.\mathbf{e}\left(\mathbf{k}_{s}, z_{a}\right)\right) d k_{x} d k_{y}=\mathbf{0}, \quad \mathbf{r}_{s} \in \text { patch } \\
\int_{-\infty}^{+\infty} \int_{-\infty}^{+\infty} \overline{\mathbf{F}}\left(\mathbf{k}_{s}, \mathbf{r}_{s}\right) \cdot\left(\overline{\mathbf{\Phi}}\left(\mathbf{k}_{s}\right) \cdot \mathbf{j}\left(\mathbf{k}_{s}\right)+\overline{\mathbf{Y}}\left(\mathbf{k}_{s}\right) .\right. \\
\left.\mathbf{e}\left(\mathbf{k}_{s}, z_{a}\right)\right) d k_{x} d k_{y}=\mathbf{0}, \quad \mathbf{r}_{s} \in \text { aperture }
\end{array}
$$

The first step in the moment method solution of Equations (8) and (9) is to expand both the patch current $\mathbf{j}\left(\mathbf{k}_{s}\right)$ and aperture field $\mathbf{E}\left(\mathbf{k}_{s}, z_{a}\right)$ as

$$
\begin{gathered}
\mathbf{j}\left(\mathbf{k}_{s}\right)=\sum_{n=1}^{N} a_{n}\left[\begin{array}{c}
J_{x n}\left(\mathbf{r}_{s}\right) \\
0
\end{array}\right]+\sum_{m=1}^{M} b_{m}\left[\begin{array}{c}
0 \\
J_{y m}\left(\mathbf{r}_{s}\right)
\end{array}\right] \\
\mathbf{E}\left(\mathbf{r}_{s}, z_{a}\right)=\sum_{p=1}^{P} c_{p}\left[\begin{array}{c}
E_{x p}\left(\mathbf{r}_{s}\right) \\
0
\end{array}\right]+\sum_{q=1}^{Q} d_{q}\left[\begin{array}{c}
0 \\
E_{y q}\left(\mathbf{r}_{s}\right)
\end{array}\right]
\end{gathered}
$$

where $J_{x n}, J_{y m}, E_{x p}$, and $E_{y q}$ are known basis functions and $a_{n}, b_{m}, c_{p}$, and $d_{q}$ are the mode expansion coefficients to be sought. Using the technique known as the moment method [17], with weighting modes chosen identical to the expansion modes, Equations (8) and (9) are reduced to a system of linear equations which can be written compactly in matrix form as

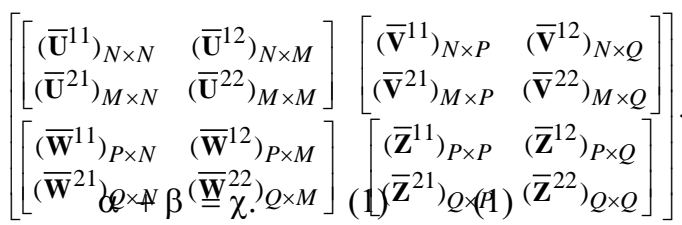

$$
\begin{aligned}
& {\left[\begin{array}{c}
(\mathbf{a})_{N \times 1} \\
(\mathbf{b})_{M \times 1} \\
(\mathbf{c})_{P \times 1} \\
\text { (d) }_{Q \times 1}
\end{array}\right]=\mathbf{0}}
\end{aligned}
$$

The elementsœf thß matrix $(\overline{\mathbf{U}})\left(\mathbb{N}_{N}\right)_{M) \times(N+M)},(\overline{\mathbf{V}})_{(N+M) \times(P+Q)}$, $(\overline{\mathbf{W}})_{(P+Q) \times(N+M)}$, and $(\overline{\mathbf{Z}})_{(P+Q) \times(P+Q)}$ are given in [13].

It is easy to show that the entire matrix in Equation (12) is a symmetric matrix. For the existence of a non-trivial solution of Equation (12), we must have

$$
\operatorname{det}(\overline{\boldsymbol{\Omega}}(f))=0, \overline{\boldsymbol{\Omega}}=\left[\begin{array}{cc}
\overline{\mathbf{U}} & \overline{\mathbf{V}} \\
\overline{\mathbf{W}} & \overline{\mathbf{Z}}
\end{array}\right]
$$

Equation $\alpha_{(13} \beta \overline{\overline{\text { is }}} \chi_{\text {the }}$ chaldeteristid) equation for the complex resonant frequency $f=f_{r}+\mathrm{i} f_{i}$ of the generalized microstrip structure illustrated in Fig.1. $f_{r}$ is the resonant frequency and $2 f_{i} / f_{r}$ is the half-power bandwidth of the structure.

In the following section, a basic artiffcial neural network is described briefly and the application of neural network to the prediction the resonant characteristics of the microstrip antenna are then explained.

\section{ARTIFICIAL NEURAL NETWORK}

Artificial neural networks (ANNs) have been successfully applied to solve many real world problems, specially the problems which can be hard tracked by expert systems. These networks canopredice the relatiddnship blettveen the input and output set without prior knowledge of the process model. The network can solve the problems related with complex engineering systems, difficult electromagnetic computation etc. [18]. In the course of developing an ANN model, the architecture of the $\beta$ neuxal netw6rk and the learning algorithm are the two most important factors. ANNs have many structures and architectures [19-20]. The class of the ANN 
and/or the architecture selected for a particular model implementation depends on the problem to be solved.

Multilayer perceptrons (MLP) have been applied successfully to solve some difficult and diverse problems by training them in a supervised manner with a highly popular algorithm known as the error back propagation algorithm [21].

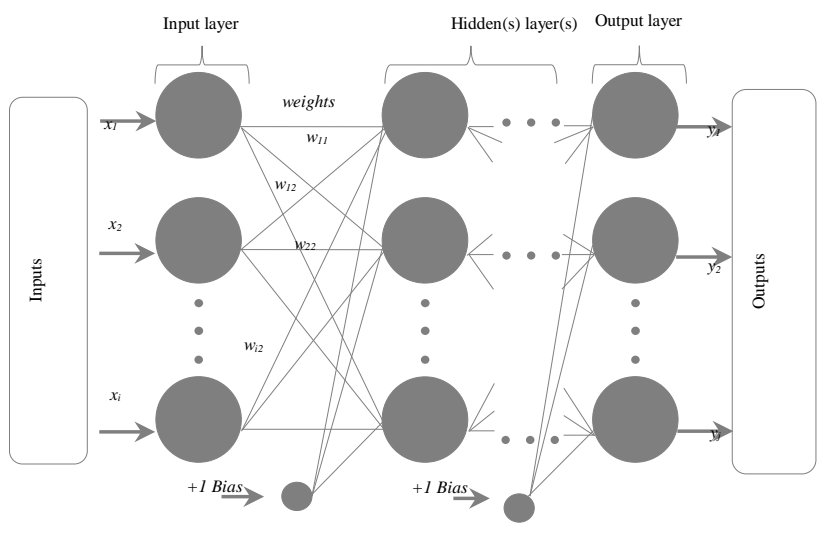

Figure 2. General form of multilayered perceptrons.

As shown in Fig.2, the MLP consists of an input layer, one or more hidden layers, and an output layer. Neurons in the input layer only act as buffers for distributing the input signals $x_{i}$ to neurons in the hidden layer. Each neuron in the hidden layer sums its input signals $x_{i}$ after weighting them with the strengths of the respective connections $w_{j i}$ from the input layer and computes its output $y_{j}$ as a function $\mathrm{f}$ of the sum, namely

$$
y_{j}=f\left(\sum w_{j i} x_{i}\right)
$$

Where $f$ can be a simple threshold function or a sigmoid or hyperbolic tangent function [22]. The output of neurons in the output layer is computed similarly. Training of a network is accomplished through adjustment of the weights to give the desired response via the learning algorithms. An appropriate structure may still fail to give a better model unless the structure is trained by a suitable learning algorithm. A learning algorithm gives the change $\Delta w_{j i}(k)$ in the weight of a connection between neurons $i$ and $j$ at time $k$. The weights are then updated according to the formula

$$
w_{j i}(k+1)=w_{j i}(k)+\Delta w_{j i}(k+1)
$$

In this work, both Multilayer Perceptron (MLP) networks were used in ANN models. MLP models were trained with almost all network learning algorithms. Hyperbolic tangent sigmoid and linear transfer functions were used in MLP training. The train and test data of the synthesis and analysis ANN were obtained from calculated with spectral model and a computer program using formula given in Section 2. The data are in a matrix form consisting inputs and target values and arranged according to the definitions of the problems. Using [19-20], two are generated for learning and testing the neural model. The different network input and output parameters are shown in Figure 3 and 4 . Some strategies are adopted to reduce time of training and ameliorate the ANN models accuracy, such as preprocessing of inputs and output, randomizing the distribution of the learning data [23], and normalized between 0.1 to 0.9 in MATLAB software before applying training. For an applied input pattern, the arbitrary numbers between 0 and 1 are assigned to initialize the weights and biases [10]. The output of the model is then calculated for that input pattern.

The CPU time taken by the spectral domain to give the both resonant frequency and half-power bandwidth for each input set is more than five minutes; it depends on three initial values used in Muller's algorithm for not seeking of the characteristic equation. All the numerical results presented in this paper we obtained on a Pentium IV computer with a 2.6$\mathrm{GHz}$ processor and a total RAM memory of $2 \mathrm{~GB}$.

In this work, the patch and aperture dimensions of the microstrip antenna are obtained as a function of input variables, which are height of the dielectric material $(d)$, dielectric constants of the substrate $\left(\varepsilon_{r}\right)$, and the resonant frequency $\left(f_{r}\right)$, using ANN techniques "Fig. 3". Similarly, in the analysis ANN, the resonant frequency of the antenna is obtained as a function of patch $\left(W_{p}, L_{p}\right)$ and aperture $\left(W_{a}, L_{a}\right)$ dimensions, height of the dielectric substrate $(d)$, and dielectric constants of the material $\left(\varepsilon_{r}\right)$ "Fig. 4". Thus, the forward and reverse sides of the problem will be defined for the circular patch geometry in the following subsections.

It should be pointed out that the presence of apertures in the ground plane $\beta_{\text {of }}$ r ficrostrip patch antennas unavoidably affects the resonant properties of the antennas. This effect of ground-plane apertures on microstrip patches has been explicitly shown in [24-25,] and [4, 17], where the authors have demonstrated that apertures in the ground plane of rectangular microstrip patches can be used as a way to tune their resonant frequencies [17]. Since ground-plane apertures can play a role in the design of microstrip patch antennas and microstrip patch circuit components. By designer point of view, it is important to give to the calculation of the antenna physical and geometrical parameters the same importance as its resonant characteristics.

Because there is no explicit model that gives the dimension of the patch (ground-ptane apertures) directly and accurately and because of the high nonlinearity of the relationship between the resonant frequency and the patch dimension (ground-plane apertures), the reverse modeling is needed [19]. Therefore, this example is very useful for illustrating features and capabilities of synthesis ANN.

\section{A. The forward side of the problem: The synthesis ANN}

The input quantities to the ANN black-box in synthesis "Fig. 3" can be ordered as: 


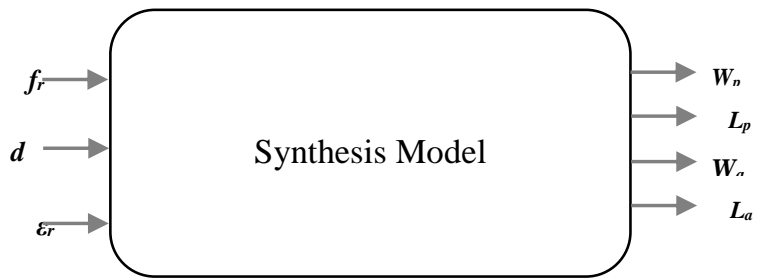

Figure 3. Synthesis Neural model for predicting the patch and aperture dimensions of an antenna with rectangular aperture in the ground plane.

- $\quad d$ : height of the dielectric substrate;

- $\varepsilon_{r}$ : effective dielectric substrate;

- $f_{r}$ : resonant frequency of the antenna.

The following quantities can be obtained from the output of the black-box as functions of the input variables:

- $W_{p}$ : width of a rectangular patch;

- $L_{p}$ : length of a rectangular patch.

- $W_{a}$ : width of a rectangular aperture;

- $\quad L_{p}$ : length of a rectangular aperture.

\section{B. The reverse side of the problem: The analysis ANN}

In the analysis side of the problem, terminology similar to that in the synthesis mechanism is used, but the resonant frequency of the antenna is obtained from the output for a chosen dielectric substrate, patch and aperture dimensions at the input side as shown in "Fig. 4".

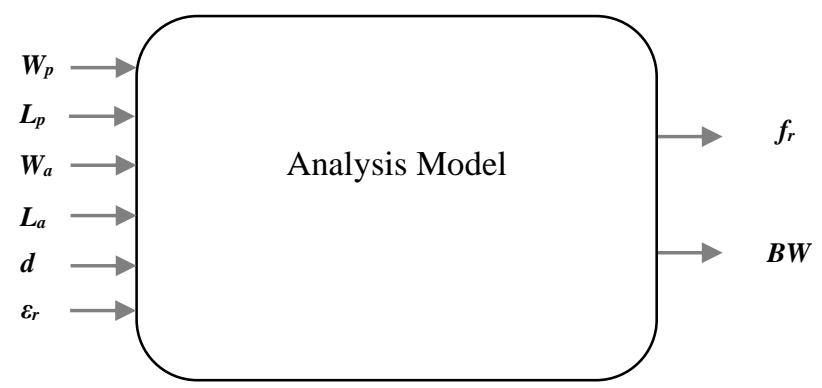

Figure 4. Analysis Neural model for predicting the resonant frequency and bandwidth of rectangular microstrip antenna with rectangular aperture in the ground plane.

To find a proper ANN-based synthesis and analysis models for rectangular microstrip antenna with rectangular aperture in the ground plane, many experiments were carried out in this study. After many trials, it was found that the target of high accuracy was summarized in Table 1.

TABLE 1. COMPARISON OF PERFORMANCE DETAILS OF ANALYSIS AND SYNTHESIS MODEL.

\begin{tabular}{|l|c|c|}
\hline \multirow{2}{*}{ Algorithm details } & \multicolumn{2}{c|}{ Neurospectral approach } \\
\cline { 2 - 3 } & Analysis model & Synthesis model \\
\hline Activation function & sigmoid & sigmoid \\
\hline Training function (back-propagation) & trainrp & trainrp \\
\hline Number of data & 250 & 250 \\
\hline Number of neurons (input layer) & 6 & 3 \\
\hline
\end{tabular}

\begin{tabular}{|l|c|c|}
\hline Number of neurons (2 hidden layers) & $12-12$ & $8-10$ \\
\hline Number of neurons (output layer) & 2 & 4 \\
\hline Epochs (number of iterations) & 5000 & 10000 \\
\hline TPE (training performance error) & $10^{-4}$ & $10^{-4}$ \\
\hline Time required & $270 \mathrm{~min}$ & $320 \mathrm{~min}$ \\
\hline LR (learning rate) & 0.6 & 0.5 \\
\hline
\end{tabular}

\section{NUMERICAL RESULTS AND DISCUSSION}

In order to determine the most appropriate suggestion given in the literature, we compared our computed values of the resonant frequencies of rectangular patch antennas with the theoretical and experimental results reported by other scientists [26], which are all given in Table 2.

From Table 3 it is observed that the bandwidths of a rectangular microstrip antenna computed by the present approach are closer to the experimental [27], and theoretical values [28-29].

TABLE 2. COMPARISON OF MEASURED AND CALCULATED RESONANT FREQUENCIES OF A RECTANGULAR MICROSTRIP ANTENNA WITH A Rectangular APERTURe IN THe GROUND Plane; $L p \times W p=34 \mathrm{~mm} \times 30 \mathrm{~mm}$, $\varepsilon_{r}=2.62$.

\begin{tabular}{|c|c|c|c|}
\hline \multirow{2}{*}{$\begin{array}{c}\text { Aperture } \\
\text { dimension } \\
\boldsymbol{L}_{\boldsymbol{a}} \times \boldsymbol{W}_{\boldsymbol{a}}\left(\mathbf{m m}^{2}\right)\end{array}$} & $\begin{array}{c}\text { Substrate thickness } \\
\boldsymbol{d}(\mathbf{m m})\end{array}$ & \multicolumn{2}{|c|}{$\begin{array}{c}\text { Resonant frequencies } \boldsymbol{f}_{\boldsymbol{r}} \\
(\mathbf{G H z})\end{array}$} \\
\cline { 3 - 4 } & & $\begin{array}{c}\text { Measured } \\
{[\mathbf{2 6}]}\end{array}$ & Our results \\
\hline $7 \times 0.7$ & 0.794 & 2.896 & 2.901 \\
\hline $10 \times 1$ & 3.175 & 2.750 & 2.770 \\
\hline
\end{tabular}

In Table 4, the resonant frequencies obtained by the present approach are compared with the previous results [30-31]. The comparison shows that the resonant frequencies computed by the present method are in very good agreement with the measured data for a rectangular patch printed on a single substrate.

TABLE 3. COMPARISON OF THE CALCULATED BANDWIDTH WITH MEASURED AND CALCULATED DATA, FOR A RECTANGULAR MiCROSTRIP PATCh ANTENNA Without APERTURe IN THE GRound Plane, $\varepsilon_{r}=2.33$.

\begin{tabular}{|c|c|c|c|c|c|c|}
\hline \multicolumn{2}{|c|}{ Input parameters $(\mathbf{m m})$} & \multicolumn{4}{|c|}{ Bandwidth (\%) } \\
\cline { 3 - 7 } & Measured & \multicolumn{3}{c|}{ Calculated } \\
\hline $\boldsymbol{W}_{\boldsymbol{p}}$ & $\boldsymbol{L}_{\boldsymbol{p}}$ & $\boldsymbol{d}$ & {$[\mathbf{2 7 ]}$} & {$[\mathbf{2 8 ]}$} & {$[\mathbf{2 9}]$} & $\begin{array}{c}\text { Our } \\
\text { results }\end{array}$ \\
\hline 57 & 38 & 3.175 & 3.12 & 4.98 & 3.5 & 3.75 \\
\hline 45.5 & 30.5 & 3.175 & 4.08 & 6.14 & 4.0 & 4.16 \\
\hline 17 & 11 & 1.524 & 6.60 & 8.21 & 4.8 & 6.70 \\
\hline
\end{tabular}

TABLE 4. COMPARISON OF CALCULATION AND MEASURED RESONANT FREQUENCIES FOR RECTANGULAR MICROSTRIP ANTENNA WiTHOUT APERTURE IN THE GROUND PLANE; WITH $L_{p}=25.08 \mathrm{~mm}$, $W_{p}=15.438 \mathrm{~mm}$. 
The results of the synthesis ANN model and comparison with the targets are given in Table 5. The very good agreement between the values obtained with the model-neuronal synthesis and target values, supports the validity of the neural model. The CPU time taken to calculate the patch dimensions (ground-plane apertures) by using synthesis model is less than a 0.09 second.

In Table 6, we compare our results obtained via the proposed neurospectral model with those obtained using the conventional spectral domain method approach (SDA). As well, to the resonant frequency and half-power bandwidth, we have also shown the CPU time in this table. It is clear that our resonant frequencies and bandwidths coincide with those obtained by the conventional moment method.

Note that, the time required for obtaining the resonant frequency and half-power bandwidth using the neurospectral model is much less in comparison to the spectral domain method.

TABLE .5 RESULTS OF THE SYNTHESIS ANN AND COMPARISON WITH THE TARGETS.

\begin{tabular}{|c|c|c|c|c|c|c|c|c|c|c|}
\hline \multicolumn{4}{|c|}{ Input parameters } & \multicolumn{3}{|c|}{ Patch dimension (mm) } & \multicolumn{4}{|c|}{ Aperture dimension $(\mathrm{mm})$} \\
\hline $\begin{array}{c}f_{r} \\
G H z)\end{array}$ & $\underset{(\mathrm{mm})}{d}$ & $\varepsilon_{r}$ & $\begin{array}{c}W- \\
\text { target }\end{array}$ & $\begin{array}{c}\boldsymbol{L}_{p^{-}} \\
\text {target }\end{array}$ & $\begin{array}{c}W_{p^{-}} \\
A N N\end{array}$ & $\begin{array}{c}L_{p^{-}} \\
A N N\end{array}$ & $\begin{array}{c}W_{a^{-}} \\
\text {target }\end{array}$ & $\begin{array}{c}L_{a^{-}} \\
\text {target }\end{array}$ & $\begin{array}{c}W_{a^{-}} \\
A N N\end{array}$ & $\begin{array}{c}\boldsymbol{L}_{a^{-}} \\
A N N\end{array}$ \\
\hline 7.30 & 0.17 & 2.22 & 8.5 & 12.9 & 8.485 & 12.91 & 1.7 & 2.6 & 1.68 & 2.60 \\
\hline 7.98 & 0.17 & 2.22 & 7.9 & 11.85 & 7.906 & 11.84 & 1.5 & 2.5 & 1.48 & 2.49 \\
\hline 3.71 & 0.79 & 2.22 & 20.0 & 25.0 & 19.980 & 24.99 & 5.0 & 7.0 & 5.05 & 7.06 \\
\hline 4.63 & 1.57 & 2.33 & 18.1 & 19.6 & 18.121 & 19.58 & 3.5 & 5.5 & 3.48 & 5.49 \\
\hline 3.96 & 3.18 & 2.33 & 29.5 & 19.5 & 19.482 & 19.49 & 6.0 & 4.0 & 6.03 & 4.02 \\
\hline 7.65 & 1.52 & 2.33 & 17.0 & 11.0 & 17.060 & 11.05 & 3.4 & 2.4 & 3.38 & 2.39 \\
\hline 2.16 & 1.52 & 2.50 & 41.4 & 41.4 & 41.385 & 41.39 & 6.2 & 6.2 & 6.19 & 6.20 \\
\hline 5.07 & 3.0 & 2.50 & 15.3 & 16.3 & 15.311 & 16.28 & 3.0 & 3.2 & 3.03 & 3.19 \\
\hline 6.34 & 2.42 & 2.55 & 11.2 & 12.0 & 11.213 & 12.08 & 5.6 & 6.0 & 5.58 & 5.98 \\
\hline 5.57 & 2.52 & 2.55 & 14.03 & 14.85 & 14.052 & 14.86 & 2.8 & 2.8 & 2.78 & 2.80 \\
\hline 4.42 & 1.27 & 10.3 & 9.1 & 10.0 & 9.114 & 10.06 & 1.8 & 2.0 & 1.81 & 1.99 \\
\hline
\end{tabular}

TABLE.6 COMPARISON OF OUR RESULTS OBTAINED VIA THE PROPOSED NEUROSPECTRAL MODEL WITH THOSE OBTAINED USING THE CONVENTIONAL SPECTRAL DOMAIN METHOD, WITH $W_{p} \times L_{p}=4 \times 2 \mathrm{~mm}^{2}$.

\begin{tabular}{|c|c|c|c|c|c|c|c|c|c|}
\hline \multicolumn{4}{|c|}{ Input parameters } & \multicolumn{3}{|c|}{$\begin{array}{c}\text { Conventional method } \\
\text { (SDA) }\end{array}$} & \multicolumn{3}{|c|}{ Neurospectral method } \\
\hline$W$ & $L$ & $d$ & \multirow{2}{*}{$\varepsilon_{r}$} & \multirow{2}{*}{$\underset{(G H z)}{f r}$} & \multirow{2}{*}{$\begin{array}{l}B w \\
(\%)\end{array}$} & \multirow{2}{*}{$\begin{array}{l}\text { CPU } \\
\text { Time } \\
\text { (min) }\end{array}$} & \multirow{2}{*}{$\underset{(G H z)}{f r}$} & \multirow{2}{*}{$\begin{array}{l}B w \\
(\%)\end{array}$} & \multirow{2}{*}{$\begin{array}{l}\text { CPU } \\
\text { Time } \\
\text { (Sec) }\end{array}$} \\
\hline \multicolumn{3}{|c|}{$(\mathrm{mm})$} & & & & & & & \\
\hline 2.5 & 2.5 & 0.6 & 2.35 & 8.956 & 3.382 & 5.39 & 8.972 & 3.365 & 0.090 \\
\hline 2.5 & 5 & 0.8 & 2.35 & 8.038 & 4.221 & 5.40 & 8.012 & 4.178 & 0.091 \\
\hline 5 & 2.5 & 1 & 2.35 & 8.710 & 5.621 & 5.43 & 8.731 & 5.642 & 0.092 \\
\hline 2.5 & 2.5 & 0.6 & 3.4 & 7.531 & 2.413 & 5.42 & 7.562 & 2.397 & 0.091 \\
\hline 2.5 & 5 & 0.8 & 3.4 & 6.784 & 2.931 & 5.40 & 6.778 & 2.894 & 0.090 \\
\hline 5 & 2.5 & 1 & 3.4 & 7.356 & 4.063 & 5.38 & 7.325 & 4.023 & 0.091 \\
\hline 2.5 & 2.5 & 1.2 & 10.3 & 4.365 & 1.394 & 5.38 & 4.352 & 1.412 & 0.091 \\
\hline 5 & 2.5 & 1.4 & 10.3 & 4.295 & 1.645 & 5.37 & 4.278 & 1.637 & 0.091 \\
\hline 2.5 & 5 & 1.8 & 10.3 & 4.057 & 2.053 & 5.41 & 4.036 & 1.997 & 0.090 \\
\hline
\end{tabular}

\section{CONCLUSION}

In this paper a general procedure is suggested for modeling and design of rectangular microstrip antenna with and without rectangular aperture in the ground plane, using spectral domain approach in conjunction with artificial neural networks. In the design stage, synthesis is defined as the forward side and then analysis as reverse side of the problem. During synthesis of the antenna, it is desirable for the design

\begin{tabular}{|c|c|c|c|c|c|}
\hline \multicolumn{2}{|c|}{ Input parameters } & \multicolumn{3}{|c|}{ Resonant frequency $\boldsymbol{f}_{\boldsymbol{r}}(\mathbf{G H z})$} \\
\cline { 3 - 6 } & Measured & \multicolumn{3}{|c|}{ Calculated } \\
\hline$d(\mathrm{~mm})$ & $\varepsilon_{r}$ & {$[30]$} & {$[30]$} & {$[31]$} & $\begin{array}{c}\text { Our } \\
\text { results }\end{array}$ \\
\hline 0.84 & 2.2 & 6.057 & 6.092 & 6.15 & 6.063 \\
\hline 1.64 & 2.2 & 5.887 & 5.883 & 5.89 & 5.885 \\
\hline
\end{tabular}

engineers to know different performance parameters of an antenna simultaneously, instead of knowing individual parameters, alternatively. Hence, the present approach has been considered more generalized and efficient. The spectral domain technique combined with the ANN method is several hundred times faster than the direct solution. This remarkable time gain makes the designing and training times negligible. Consequently, the neurospectral method presented in this paper is a useful method that can be integrated into a CAD tool, for the analysis, design, and optimization of practical shielded (Monolithic microwave integrated circuit) MMIC devices.

\section{REFERENCES}

[1] K. Guney and N. Sarikaya, "A hybrid method based on combining artificial neural network and fuzzy inference system for simultaneous computation of resonant frequencies of rectangular, circular, and triangular microstrip antennas," IEEE Transactions on Antennas and Propagation, vol. 55, pp. 659-668, 2007.

[2] A. Kalinli, S. Sagiroglu, and F. Sarikoc, "Parallel ant colony optimization algorithm based neural method for determining resonant frequencies of various microstrip antennas," Electromagnetics, vol. 30, pp. 463-481, 2010.

[3] I. Vilovic, N. Burum, and M. Brailo, "Microstrip antenna design using neural networks optimized by PSO," 21st International Conference in Applied Electromagnetics and Communications (ICECom), 2013, pp. 14.

[4] T. Fortaki, D. Khedrouche, F. Bouttout, and A. Benghalia, "Numerical analysis of rectangular microstrip patch over ground plane with rectangular aperture," Communications in numerical methods in engineering, vol. 20, pp. 489-500, 2004.

[5] M.-H. Ho and C.-I. Hsu, "Circular-waveguide-fed microstrip patch antennas," Electronics Letters, vol. 41, pp. 1202-1203, 2005.

[6] A. Verma and Nasimuddin, "Multilayer Cavity Model for Microstrip Rectangular and Circular Patch Antenna," Electromagnetics, vol. 24, pp. 193-217, 2004.

[7] C. Gürel and E. Yazgan, "Resonant frequency of air gap tuned circular microstrip antenna with anisotropic substrate and superstrate layers," Journal of Electromagnetic Waves and Applications, vol. 24, pp. 17311740, 2010.

[8] S. Bedra, S. Benkouda, and T. Fortaki, "Analysis of a circular microstrip Antenna on Isotropic or uniaxially anisotropic substrate Using neurospectral approach," COMPEL: The International Journal for Computation and Mathematics in Electrical and Electronic Engineering, vol. 33, pp. 41-41, 2013.

[9] D. Guha and Y. M. Antar, Microstrip and printed antennas: new trends, techniques and applications: John Wiley \& Sons, 2011.

[10] T. Khan, A. De, and M. Uddin, "prediction of slot-size and inserted airgap for improving the performance of rectangular microstrip antennas using artificial neural networks," IEEE Antennas and Wireless Propagation Letters, vol. 12, pp. 1367-1371, 2013.

[11] S. K. Jain, A. Patnaik, and S. N. Sinha, "Design of custom-made stacked patch antennas: a machine learning approach," International Journal of Machine Learning and Cybernetics, vol. 4, pp. 189-194, 2013.

[12] W. Zhongbao, F. Shaojun, W. Qiang, and L. Hongmei, "An ANN-Based Synthesis Model for the Single-Feed Circularly-Polarized Square Microstrip Antenna With Truncated Corners," IEEE Transactions on Antennas and Propagation, vol. 60, pp. 5989-5992, 2012. 
[13] M. Aneesh, A. Singh, J. A. Ansari, and S. S. Sayeed, "Investigations for Performance Improvement of X-Shaped RMSA Using Artificial Neural Network by Predicting Slot Size," Progress in Electromagnetics Research C, vol. 47, 2014.

[14] T. Bose and N. Gupta, "Design of an aperture-coupled microstrip antenna using a hybrid neural network," IET Microwaves, Antennas \& Propagation, ,vol. 6, pp. 470-474, 2012.

[15] R. Mishra and A. Patnaik, "Neurospectral computation for complex resonant frequency of microstrip resonators," IEEE Microwave and Guided wave letters, vol. 9, pp. 351-353, 1999.

[16] R. Mishra and A. Patnaik, "Neurospectral computation for input impedance of rectangular microstrip antenna," Electronics Letters, vol. 35, pp. 1691-1693, 1999.

[17] T. Fortaki and A. Benghalia, "Rigorous full- wave analysis of rectangular microstrip patches over ground planes with rectangular apertures in multilayered substrates that contain isotropic and uniaxial anisotropic materials," Microwave and Optical Technology Letters, vol. 41, pp. 496-500, 2004

[18] P. Samaddar, S. Nandi, S. Nandy, D. Sarkar, and P. Sarkar, "Prediction of resonant frequency of a circular patch frequency selective structure using artificial neural network," Indian Journal of Physics, Vol. 88, pp. 397-403, 2014.

[19] Y. Tighilt, F. Bouttout, and A. Khellaf, "Modeling and design of printed antennas using neural networks," International Journal of RF and Microwave Computer- Aided Engineering, vol. 21, pp. 228-233, 2011.

[20] C. Christodoulou and M. Georgiopoulos, Applications of neural networks in electromagnetics: Artech House, Inc., 2000.

[21] K. Kumar and N. Gunasekaran, "Bandwidth enhancement of a notch square shaped microstrip patch antenna using neural network approach," International Conference in Emerging Trends in Electrical and Computer Technology (ICETECT), Tamil Nadu 2011, pp. 797-799.
[22] K. Guney and S. Gultekin, "A comparative study of neural networks for input resistance computation of electrically thin and thick rectangular microstrip antennas," Journal of Communications Technology and Electronics, vol. 52, pp. 483-492, 2007.

[23] Z. Raida, "Modeling EM structures in the neural network toolbox of MATLAB," IEEE Antennas and Propagation Magazine, vol. 44, pp. 4667, 2002.

[24] K. Kawano and H. Tomimuro, "Hybrid-mode analysis of a microstripslot resonator," in IEE Proceedings $\mathrm{H}$ (Microwaves, Optics and Antennas),Vol. 129 , pp. 351-355, 1982.

[25] K. Kawano, "Hybrid-mode analysis of coupled microstrip-slot resonators," IEEE Transactions on Microwave Theory and Techniques, vol. 33, pp. 38-43, 1985 .

[26] M. I. Aksun, S.-L. Chuang, and Y. T. Lo, "On slot-coupled microstrip antennas and their applications to CP operation-theory and experiment," IEEE Transactions on Antennas and Propagation, vol. 38, pp. 12241230,1990

[27] E. Chang, S. A. Long, and W. F. Richards, "An experimental investigation of electrically thick rectangular microstrip antennas," IEEE transactions on antennas and propagation, vol. 34, pp. 767-772, 1986.

[28] W. C. Chew and Q. Liu, "Resonance frequency of a rectangular microstrip patch," IEEE Transactions on Antennas and Propagation, vol. 36, pp. 1045-1056, 1988.

[29] D. M. Pozar: PCAAD 3.0. Personal Computer Aided Antenna Design, Antenna Design Associates, Inc 1996

[30] S. Chattopadhyay, M. Biswas, J. Y. Siddiqui, and D. Guha, "Rectangular microstrips with variable air gap and varying aspect ratio: improved formulations and experiments," Microwave and Optical Technology Letters, vol. 51, pp. 169-173, 2009.

[31] HFSS: High Frequency Structure Simulator, Ansoft Corp., 2009. 